\title{
Spin-fluctuation-induced pairing in twisted bilayer graphene
}

\author{
Ammon Fischer $\odot,{ }^{1}$ Lennart Klebl $\odot,{ }^{2}$ Carsten Honerkamp, ${ }^{1}$ and Dante M. Kennes ${ }^{2,3}$ \\ ${ }^{1}$ Institute for Theoretical Solid State Physics, RWTH Aachen University, and JARA Fundamentals of Future Information Technology, \\ 52062 Aachen, Germany \\ ${ }^{2}$ Institute for Theory of Statistical Physics, RWTH Aachen University, and JARA Fundamentals of Future Information Technology, \\ 52062 Aachen, Germany \\ ${ }^{3}$ Max Planck Institute for the Structure and Dynamics of Matter, Center for Free Electron Laser Science, 22761 Hamburg, Germany
}

(Received 28 August 2020; revised 12 December 2020; accepted 15 December 2020; published 7 January 2021)

\begin{abstract}
We investigate the interplay of magnetic fluctuations and Cooper pairing in twisted bilayer graphene from a purely microscopic model within a large-scale tight-binding approach resolving the angstrom scale. For local on-site repulsive interactions and using the random-phase approximation for spin fluctuations, we derive a microscopic effective pairing interaction that we use for self-consistent solutions of the Bogoliubov-de Gennes equations of superconductivity. We study the predominant pairing types as a function of interaction strength, temperature, and band filling. For large regions of this parameter space, we find chiral $d$-wave pairing regimes, spontaneously breaking time-reversal symmetry, separated by magnetic instabilities at integer band fillings. Interestingly, the $d$-wave pairing is strongly concentrated in the $A A$ regions of the moire unit cell and exhibits phase windings of integer multiples of $2 \pi$ around these superconducting islands, i.e., pinned vortices. The spontaneous circulating current creates a distinctive magnetic field pattern. This signature of the chiral pairing should be measurable by state-of-the-art experimental techniques.
\end{abstract}

DOI: 10.1103/PhysRevB.103.L041103

Introduction. Twisted two-dimensional materials have become an extremely active research area, with twisted bilayer [1-17], trilayer [18] and double bilayer graphene [19-25], transition metal dichalcogenides homo- and heterobilayers [26-29] as well as other materials [30-32] at the frontier of condensed matter research. These systems are fascinating due to a high degree of band-structure and correlation engineering that can be achieved, putting unprecedented topological and exotic correlated states within experimental reach [33]. In fact, twisting can lead to strong alterations of the band structures, most notably by the formation of flatbands, that may enhance various interaction effects and hence lead to interesting and potentially novel, interaction-driven ground states [33]. From a theoretical point of view, the twisting-induced large moiré unit cell poses a formidable challenge for the description on the atomic scale as an excessive amount of degrees of freedom have to be treated.

With regard to twisted bilayer graphene (TBG), which initially triggered these immense research activities on twisted two-dimensional (2D) heterostructures, the main questions about the quantum many-body state at low temperatures still concerns the nature of the insulating and superconducting states found experimentally. Recently, we have addressed the question in the context of insulating states by microscopic random-phase approximation (RPA) [34] and functional renormalization group (fRG) techniques [35], keeping the full unit cell containing many thousands of atoms (depending on the twist angle) under microscopic scrutiny. This microscopic theory on the carbon-carbon bond scale predicts the leading electronic interaction-driven instabilities for a larger parameter range to be magnetically ordered with strong spatial variations of the order parameter through the large moiré unit cell. However, in the same approach, superconducting pairing instabilities have not received much attention, beyond a rescaled theory losing some of the connection to the microscopics [36-38].

Thus, despite the enormous theoretical effort in the young history of this field, fully microscopic models that capture the full electronic spectrum of TBG while providing a mechanism for strong electron-electron interaction are still rare. In this Letter, we remedy this shortcoming by (i) using a full tightbinding approach for the $\pi$ bands of TBG, (ii) deriving an effective two-particle interaction vertex $\Gamma_{2}$ by using the RPA to include spin-fluctuation exchange between electrons to high orders in the bare couplings, and (iii) using a mean-field decoupling of this effective interaction to analyze the nature and experimental signatures of the favored superconducting state in a fully microscopic model. By considering spin fluctuations alone as the potential pairing glue we do neglect other forms of electronic two-particle scattering. When considering on-site bare electron-electron interactions only, this approximation was recently shown to be justified comparing RPA and unbiased fRG techniques [34,35], but of course including phonons would widen the range of possibilities beyond this study. From the derived electronically mediated pairing interaction we extract pairing symmetries and the spatial distribution of the superconducting order parameter on the carbon-carbon bond scale, with the advantage of this approach being that it allows one to study both magnetic instabilities and superconducting pairing as a function of temperature and 
doping. Our work thus establishes a microscopic scenario of electronic correlation effects leading to the phase diagrams of TBG recently measured in experiments $[1,3,5]$ and provides direct predictions to be tested. As a function of the carrier density, between the insulating states at integer fillings, we find an attractive spin-fluctuation mediated pairing vertex that gives rise to chiral $d$-wave pairing regimes, spontaneously breaking time-reversal symmetry (TRS). The superconducting order parameter is strongly enhanced in the $A A$ regions of the system and exhibits supercurrents within a vortexantivortex structure as well as magnetic fields, which yield measurable signatures of the chiral pairing in experiment. This will help to distinguish the origin of superconductivity in TBG and settle the debate about whether its mechanism is electron or phonon driven [4].

Methods. For our fully microscopic theory we first follow Ref. [39] to set up an atomistic tight-binding Hamiltonian for the $\pi$-band spectrum of magic-angle TBG $\left(\theta=1.05^{\circ}\right)$ keeping all $N=11908$ bands under consideration:

$$
H_{0}=\sum_{\boldsymbol{R}, \boldsymbol{R}^{\prime}} \sum_{i, j, \sigma} t\left(\boldsymbol{R}+\boldsymbol{r}_{i}-\boldsymbol{R}^{\prime}-\boldsymbol{r}_{j}\right) c_{\boldsymbol{R}, \boldsymbol{r}_{i}, \sigma}^{\dagger} c_{\boldsymbol{R}^{\prime}, \boldsymbol{r}_{j}, \sigma} .
$$

The hopping parameters decay exponentially on the carboncarbon bond scale (see the Supplemental Material [40] for details) and are derived from first-principles calculations [39]. When taking atomic relaxation effects between the layers into account, the spectrum contains four flat bands (twofold spin degenerate) around charge neutrality, separated from the rest of the spectrum.

We include interaction effects by a repulsive Hubbard term for electrons with opposite spin $\sigma$ residing on the same carbon site:

$$
H_{\mathrm{int}}=\frac{1}{2} \sum_{\boldsymbol{R}, i, \sigma} U n_{\boldsymbol{R}, \boldsymbol{r}_{i}, \sigma} n_{\boldsymbol{R}, \boldsymbol{r}_{i}, \bar{\sigma}}
$$

where $\boldsymbol{R}$ labels the supercell vector and $\boldsymbol{r}_{i}$ is restricted to the moire unit cell. This on-site term is an idealization of the true long-ranged nature of the actual Coulomb interaction. Theoretical work for nontwisted systems [41,42] indicates that the main instabilities towards insulating states are correctly captured by this idealization. The value for $U$ is well established within the constrained RPA approximation for monoand bilayer graphene $[43,44]$ and the effect of the nonlocal terms may be absorbed into an effective $U^{*}$ [45].

To characterize the potential ground state of the interacting system, a two-step protocol is employed. First, we study spin fluctuations and associated magnetic ordering of TBG by using the RPA for the magnetic susceptibility $\hat{\chi}(\boldsymbol{q}, v)$. Here, we exploit the methodology proposed in Ref. [34] that captures spin fluctuations on the carbon-carbon bond scale with emphasis on the static, long-wavelength limit $(\boldsymbol{q}, v \rightarrow 0)$ on the moiré scale. The latter limit proves to contain the relevant physics when starting with local repulsive interaction. The RPA susceptibility predicts spin correlations at length scales intermediate to the $\mathrm{C}-\mathrm{C}$ bond scale and moiré length scale, thus being described by orderings at $\boldsymbol{q}=0$. The system shows the same order in all moiré unit cells with variable correlations present on the $\mathrm{C}-\mathrm{C}$ bond scale.

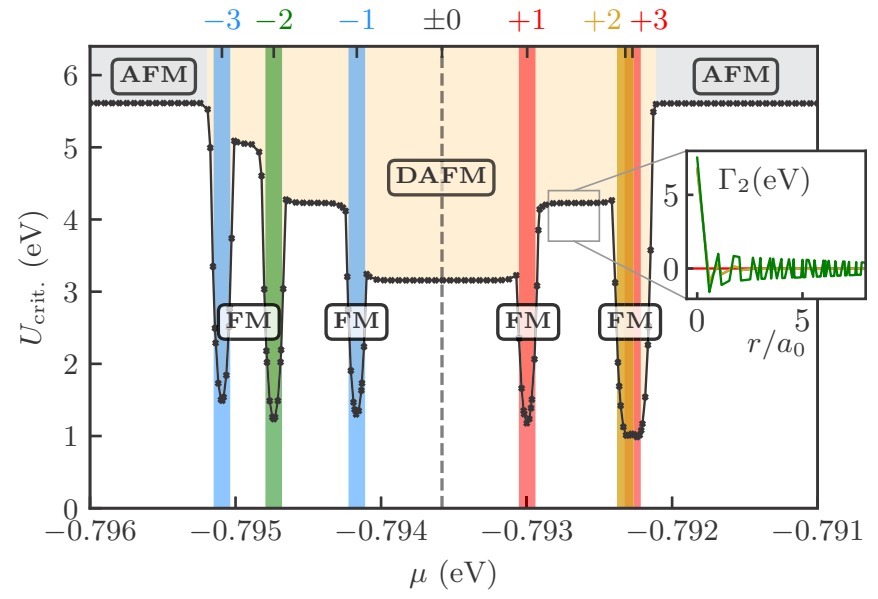

FIG. 1. Magnetic RPA phase diagram showing the critical on-site interaction strength $U_{\text {crit. }}$ vs chemical potential $\mu$ in the four flatbands of TBG at $T=0.03 \mathrm{meV}$. The vertical lines indicate the integer fillings $\pm 3, \pm 2, \pm 1$ that show an increased magnetic ordering tendency towards a ferromagnetic state, while away from integer fillings weaker antiferromagnetic tendencies dominate. The boxed abbreviations indicate the type of magnetic ordering: FM, ferromagnetic order; AFM, antiferromagnetic order; DAFM, antiferromagnetic order with real-space node [34]. The spin-fluctuation interaction (inset) is attractive on nearest-neighbor bonds close to a DAFM instability, while it is purely repulsive near a FM instability.

In a second step, we deduce an effective singlet pairing vertex $\hat{\Gamma}_{2}(\boldsymbol{q}, v)$ from transverse and longitudinal spin fluctuations $[46,47]$

$$
\hat{\Gamma}_{2}(\boldsymbol{q}, v)=\hat{U}-\frac{U^{2} \hat{\chi}_{0}(\boldsymbol{q}, v)}{1+U \hat{\chi}_{0}(\boldsymbol{q}, v)}+\frac{U^{3} \hat{\chi}_{0}^{2}(\boldsymbol{q}, v)}{1-U^{2} \hat{\chi}_{0}^{2}(\boldsymbol{q}, v)},
$$

and analyze the vertex by using a mean-field decoupling to extract pairing symmetries and spatial distribution of the superconducting order parameter in the moiré unit cell. Again, we neglect the momentum dependence of the interaction vertex and thus focus on the pairing structure on the $\mathrm{C}-\mathrm{C}$ bonds within the moiré unit cell.

Magnetic instabilities. It is instructive to first analyze the magnetic ordering tendencies suggested by the RPA analysis. The instabilities can be classified according to a generalized Stoner criterion: the effective interaction Eq. (3) diverges, when an eigenvalue of $\hat{\chi}_{0}$ reaches $-1 / U$ [for details see Supplemental Material (SM) [40]]. The corresponding eigenvector yields information about the spatial structure of orbital magnetization in the moiré unit cell [48]. The key result is the phase diagram in Fig. 1. It shows the critical interaction strength $U_{\text {crit }}$ as a function of the chemical potential $\mu$ tuned in the four flat bands of TBG. The temperature $T=0.03 \mathrm{meV}$ is fixed for all simulations. For the sake of brevity, we here adapt the classification taken in Ref. [34] and label the different leading eigenvectors of $\hat{\chi}_{0}(0,0)$ according to their real-space profile: FM, ferromagnetic order; AFM, antiferromagnetic order; DAFM, (domain wall) antiferromagnetic order with real-space node (shown in the SM [40]). Interestingly, the system shows an increased magnetic ordering tendency towards a ferromagnetic ordered state when the chemical potential is fine-tuned to one of the integer fillings $\pm 3, \pm 2, \pm 1$. For 
partially filled bands, weaker antiferromagnetic patterns prevail. Our results seem to reinforce recent conductance measurements [3,24] predicting Mott insulatorlike behavior at charge neutrality and particle-hole asymmetric insulating states at integer fillings. We stress that our eigenvector analysis is not suitable to make any direct quantitative predictions about band gaps in the electronic spectrum. Nevertheless, the separation of the flatbands from the rest of the spectrum as well as the robustness with the low $U_{\text {crit }}$ at integer fillings suggest that for these dopings, the ordering should lead to a complete splitting of the flatbands and thus insulating states.

Unconventional superconductivity. Next, we proceed with analyzing the spin-fluctuation-induced pairing interaction Eq. (3) in the static, long-wavelength limit. At integer fillings $\pm 3, \pm 2, \pm 1$, the FM magnetic instability clearly dominates with rather low critical interaction strengths, and pairing may not be relevant. In between the integer fillings, the DAFM instability dominates the magnetic channel but, depending on the interaction value, may not be strong enough to actually occur. Then, below the threshold for the DAFM instability, the attractive pairing channels contained in the effective spinmediated pairing vertex $\hat{\Gamma}_{2}$ may induce pairing instabilities at sufficiently low $T$, consistent with experimental measurements, where superconducting regions appear between correlated insulator states located at integer fillings $[1,3]$. The real-space dependence of $\hat{\Gamma}_{2}$ is shown in the inset of Fig. 1. It is staggered throughout the moiré unit cell, including strong on-site repulsion and nearest-neighbor attraction. Such an alternating interaction is known to allow for pairing interactions for unconventional singlet Cooper pairs living on the bonds bridging the sign changes [49]. On the honeycomb lattice for these band fillings, it is expected to drive spin-singlet $d$-wave pairing states on nearest-neighbor bonds [50,51], which would now occur in the two graphene sheets of TBG. The amplitude of the effective interaction decays exponentially on the C-C bond scale and is largest in the $A A$ regions with only minor contributions in the $A B(B A)$ and domain wall (DW) regions. The same applies for the interlayer interaction, although, importantly, the latter is an order of magnitude smaller than comparable intralayer terms. This indicates that the main pairing will create in-plane Cooper pairs. As the interaction term diverges in the limit $U \rightarrow U_{\text {crit }}$, we can effectively control the overall amplitude of $\hat{\Gamma}_{2}$ by tuning the only free parameter $U$ of our model.

Based on this argumentation, as a direct consequence of the proximity to a moiré-modulated antiferromagnetic state, we now restrict our mean-field decoupling to spin-singlet configurations

$$
\begin{aligned}
\Delta_{n m}(\boldsymbol{k})= & -\frac{1}{2 N} \sum_{\boldsymbol{k}^{\prime} \sigma} \Gamma_{2, n m}\left(\boldsymbol{q}=\boldsymbol{k}-\boldsymbol{k}^{\prime}, 0\right) \\
& \times \sigma\left\langle c_{n \sigma}\left(\boldsymbol{k}^{\prime}\right) c_{m \bar{\sigma}}\left(-\boldsymbol{k}^{\prime}\right)\right\rangle_{\mathrm{MF}}
\end{aligned}
$$

where $\sigma= \pm 1$ is the spin index and $\bar{\sigma}=-\sigma$. Based on the fast decay of the pairing interaction on the moiré scale, we can assume that the momentum dependence of the gap function in Eq. (4) is rather weak when viewed in the small moiré Brillouin zone. The mentioned $d$-wave pairing will then be encoded in the $n m$ dependence. Dropping the $\boldsymbol{k}$-dependence, we solve the resulting Bogoliubov-de Gennes equations self-consistently in Nambu space [52]. To ensure proper convergence of this large-scale self-consistent calculation, we track the free energy $F \approx-\sum_{n} E_{n}-\sum_{n m}\left|\Delta_{n m}\right|^{2} / \Gamma_{2, n m}$ during each self-consistent cycle and compare the converged results for different initial gaps (see SM). In order to characterize the superconducting order parameter with respect to different pairing channels, we project $\Delta_{n m}$ onto the real-space form-factor basis of the original lattice of carbon atoms,

$$
\Delta_{\eta}(n)=\sum_{l} f_{\eta}\left(\boldsymbol{\delta}_{l}\right)\left(c_{n \uparrow} c_{n+\boldsymbol{\delta}_{l} \downarrow}-c_{n \downarrow} c_{n+\boldsymbol{\delta}_{l} \uparrow}\right),
$$

where $\eta$ denotes different pairing channels: $s^{+}, d_{x y}$, and $d_{x^{2}-y^{2}}$. The form factors $f_{\eta}\left(\boldsymbol{\delta}_{l}\right)$ are specific to the pairing channel and result from symmetrizing the bond functions $\boldsymbol{\delta}_{l}$ with the irreducible representations of the point group $D_{6 h}$ of graphene [53]. Here, we only take the $l=1,2,3$ nearestneighbor $\mathrm{C}-\mathrm{C}$ bonds $\boldsymbol{\delta}_{l}$ into account, as they are dominant in terms of attractive interaction strength.

First, we study the dependence of the order parameter $\Delta_{n m}$ projected on $s$ and $d$ waves as a function of the distance to the critical on-site interaction strength $U_{\text {crit }}$ for fixed chemical potential $\mu=-0.7945 \mathrm{eV}$, i.e., between fillings -2 and -1 and representative for the fillings where stronger $U$ would cause a DAFM instability. When varying $U$, the system makes a first-order phase transition from a "chiral" superconducting phase to a striped phase at $U_{\text {crit }}-U \approx 0.04 \mathrm{eV}$ as depicted in Fig. 2. In the chiral phase, the system assumes pure $d+i d$ order on the carbon-carbon bond scale, spontaneously breaking TRS. The order parameter is strongly enhanced in the $A A$ regions, forming superconducting islands that maintain the original $C_{3}$ symmetry of the normal-state Hamiltonian on the moiré scale. The amplitude is suppressed and vanishes completely in the $A B(B A)$ regions. At the same time, the phase of the superconducting gap exhibits windings of $2 \pi$ around these regions, giving rise to a vortex-antivortex structure with distinctive signatures in the bond current

$$
\boldsymbol{J}_{n m}=\frac{e}{i \hbar}\left\langle c_{n}^{\dagger} t_{n m} c_{m}-c_{m}^{\dagger} t_{m n} c_{n}\right\rangle \hat{\boldsymbol{e}}_{n m} .
$$

The "striped" phase, however, is characterized by a real $\Delta_{n m}$ and thus restores TRS, but breaks the original $C_{3}$ symmetry on the moiré scale. The $s^{+}$component jumps from zero to a finite value and therefore the phase is of type $s+d$ which was also mentioned in Ref. [36]. No vortex structures occur in this phase. As the striped phase breaks $C_{3}$ symmetry but not translational symmetry, it represents a nematic superconducting phase that has been found experimentally in Ref. [54]. In both phases, we observe a phase shift of $\pi$ between the two graphene sheets as a result of the original interlayer repulsion.

To further analyze the vortex structure appearing in the chiral superconducting phase of the system, we compute the layer-resolved quasiparticle bond current [52] in TBG. The current vector field $\boldsymbol{J}$ shown in Fig. 3 results from averaging the bond-restricted term $\boldsymbol{J}_{n m}$ over nearest-neighbor sites. The pattern indicates a ring current around the $A A$ regions with vanishing amplitude in the center. The same holds for the $A B(B A)$ and DW regions. The current corotates in the two graphene sheets, which thus have the same vorticity. In general, the direction of rotation assumed by the system depends on the initial guess for $\Delta_{n m}$ as expected in the context 


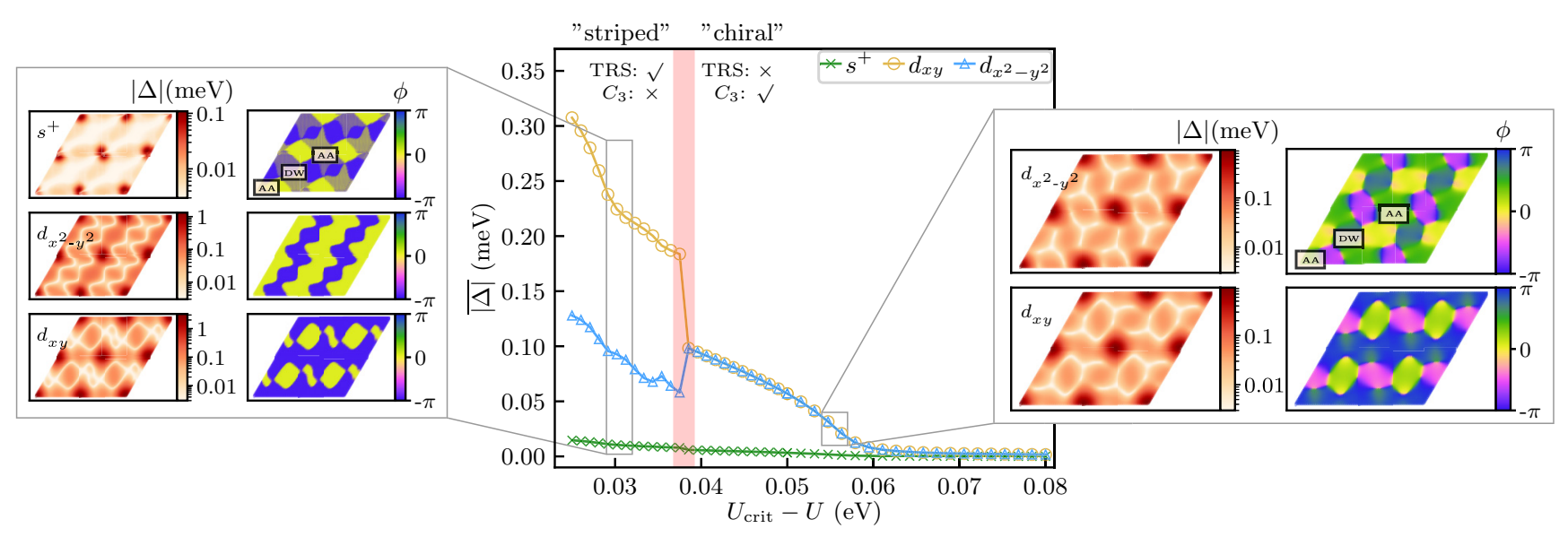

FIG. 2. Averaged amplitude of the superconducting order parameter $\overline{|\Delta|}$ projected onto the form factor basis of nearest-neighbor bonds $s^{+}$, $d_{x^{2}-y^{2}}$, and $d_{x y}$ of the original C-atom graphene lattice, as a function of the repulsive Hubbard $U$ for $\mu=-0.7945 \mathrm{eV}$ and $T=0.03 \mathrm{meV}$. The left panel shows the amplitude and phase distribution of the striped superconducting phase in the lower layer of TBG, while the right panel depicts the "chiral phase. The amplitude is strongly enhanced in the $A A$ regions forming superconducting islands, but vanishes in the $A B$ ( $B A$ ) regions.

of spontaneous symmetry breaking. When starting with timereversed initial gaps (suggesting an inverted current) in the two layers, the system converges into a state with higher free energy such that corotation is energetically favored and the magnetic fields created by the supercurrents in both layers add constructively.

In total, we identify six (anti-)vortices per moiré unit cell: one vortex in the $A A$ region forming a triangular lattice, two vortices in the $A B$ region (honeycomb lattice), and three antivortices in the DW regions (kagome lattice); see linecut of Fig. 3 for the direction of rotation. Thus, the total vorticity

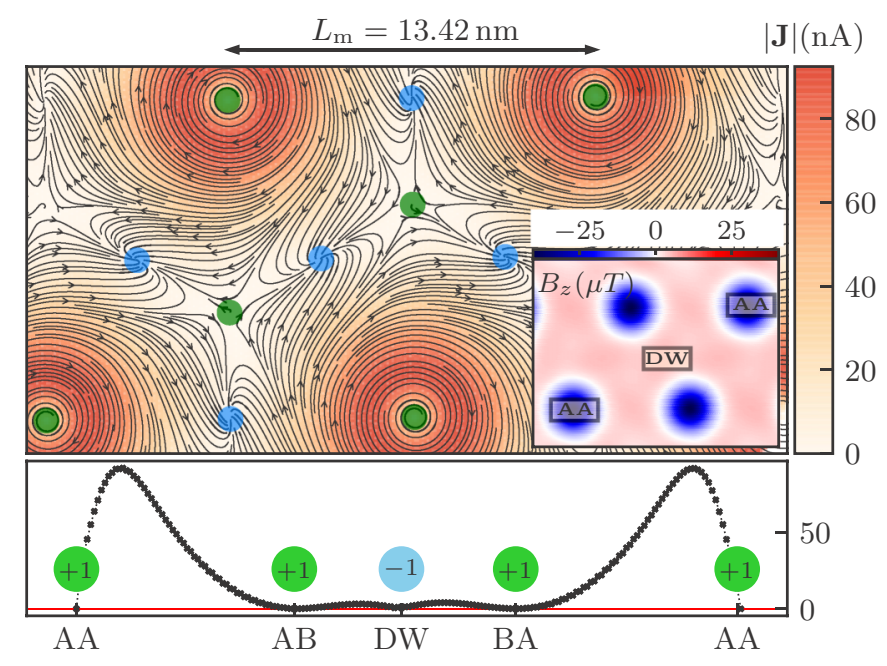

FIG. 3. Magnitude and direction of the bond current $\boldsymbol{J}_{n m}$ in the lower layer of TBG for $\mu=-0.7945 \mathrm{eV}, T=0.03 \mathrm{meV}$ in the chiral superconducting phase of the system. The current pattern indicates vortices in the $A A, A B(B A)$, and DW regions with the vorticity being labeled in the linecut through the moiré unit cell in the lower panel. The ring current is strongest in the $A A$ regions and the vorticity averages to zero over the moiré unit cell. The inset shows the magnetic field induced by the supercurrent at a distance corresponding to the interlayer spacing of TBG. is zero in the moiré unit cell. However, the vortices on the triangular lattice of the $A A$ sites dominate the current signal entirely due to the larger order parameter in these regions and the other vortices might not be easily found experimentally.

The spontaneously flowing currents induce a magnetic field that can be calculated by applying Biot-Savart's law. According to our calculations, the magnetic field is of the order of $\mu \mathrm{T}$ at a distance corresponding to the spacing between the layers. Therefore, the spontaneously formed, current-induced magnetic field is within experimental reach of state-of-the-art techniques [55]. Due to aforementioned dominant current amplitude around the $A A$ regions, the more subtle (anti-)vortex structure beyond the $A A$ region will be very difficult to resolve in magnetic measurements.

Conclusion. We investigated a theoretical scenario in which the superconducting regions found in twisted bilayer graphene are due to electronically mediated paring, in a microscopic model that resolves the carbon-carbon bond length and captures the full $\pi$ bandwidth of the layers. To this end, we used an ab-initio-based band structure of magic-angle TBG, keeping the many thousand atoms in the unit cell within our modeling. We derived a spin-fluctuation-mediated interaction vertex from these microscopic grounds. The phase diagram suggests correlated magnetic and insulating states at integer fillings and attractive electron-mediated interactions between these fillings, giving rise to unconventional pairing states consistent with recent measurements. A successive mean-field decoupling revealed an inhomogeneous, chiral order parameter on the carbon-carbon scale that is strongly enhanced in the $A A$ region. It exhibits spontaneous supercurrents and magnetic fields in certain parameter regimes that should be measurable signatures in experiment. This shows an exciting extension of the complexity of unconventional superconducting states in these systems: besides breaking the point group symmetry and time reversal by a gap function that winds in wave-vector space, the gap function in our case varies strongly and also winds in real space within the moiré unit cell, due to the chirality of the underlying bond pairing. 
As next steps, we suggest the experimental scrutiny of the phase-winding signatures of the electronically mediated pairing. These should be addressable by the currents and consequently magnetic field induced. In theory, one should include phonon-mediated interaction in the angstrom model, e.g., obtained from ab initio calculations. This will reveal the interplay and importance of different potential origins of superconductivity [4], which is a highly anticipated avenue of future research.
Acknowledgments. The Deutsche Forschungsgemeinschaft (DFG, German Research Foundation) is acknowledged for support through RTG 1995 and under Germany's Excellence Strategy - Cluster of Excellence Matter and Light for Quantum Computing (ML4Q) EXC 2004/1-390534769. We acknowledge support from the Max Planck-New York City Center for Non-Equilibrium Quantum Phenomena. Simulations were performed with computing resources granted by RWTH Aachen University under project rwth0554.
[1] Y. Cao, V. Fatemi, S. Fang, K. Watanabe, T. Taniguchi, E. Kaxiras, and P. Jarillo-Herrero, Nature (London) 556, 43 (2018).

[2] M. Yankowitz, S. Chen, H. Polshyn, Y. Zhang, K. Watanabe, T. Taniguchi, D. Graf, A. F. Young, and C. R. Dean, Science 363, 1059 (2019).

[3] X. Lu, P. Stepanov, W. Yang, M. Xie, M. A. Aamir, I. Das, C. Urgell, K. Watanabe, T. Taniguchi, G. Zhang et al., Nature (London) 574, 653 (2019).

[4] P. Stepanov, I. Das, X. Lu, A. Fahimniya, K. Watanabe, T. Taniguchi, F. H. L. Koppens, J. Lischner, L. Levitov, and D. K. Efetov, Nature (London) 583, 375 (2020).

[5] Y. Cao, V. Fatemi, A. Demir, S. Fang, S. L. Tomarken, J. Y. Luo, J. D. Sanchez-Yamagishi, K. Watanabe, T. Taniguchi, E. Kaxiras et al., Nature (London) 556, 80 (2018).

[6] A. L. Sharpe, E. J. Fox, A. W. Barnard, J. Finney, K. Watanabe, T. Taniguchi, M. A. Kastner, and D. GoldhaberGordon, Science 365, 605 (2019).

[7] M. Serlin, C. Tschirhart, H. Polshyn, Y. Zhang, J. Zhu, K. Watanabe, T. Taniguchi, L. Balents, and A. Young, Science 367, 900 (2020).

[8] C. Xu and L. Balents, Phys. Rev. Lett. 121, 087001 (2018).

[9] C.-C. Liu, L.-D. Zhang, W.-Q. Chen, and F. Yang, Phys. Rev. Lett. 121, 217001 (2018).

[10] D. M. Kennes, J. Lischner, and C. Karrasch, Phys. Rev. B 98, 241407(R) (2018).

[11] G. Li, A. Luican, J. M. B. Lopes dos Santos, A. H. Castro Neto, A. Reina, J. Kong, and E. Y. Andrei, Nat. Phys. 6, 109 (2010).

[12] A. Kerelsky, L. J. McGilly, D. M. Kennes, L. Xian, M. Yankowitz, S. Chen, K. Watanabe, T. Taniguchi, J. Hone, C. Dean et al., Nature (London) 572, 95 (2019).

[13] Y. Xie, B. Lian, B. Jäck, X. Liu, C.-L. Chiu, K. Watanabe, T. Taniguchi, B. A. Bernevig, and A. Yazdani, Nature (London) 572, 101 (2019).

[14] Y. Jiang, X. Lai, K. Watanabe, T. Taniguchi, K. Haule, J. Mao, and E. Y. Andrei, Nature (London) 573, 91 (2019).

[15] Y. Choi, J. Kemmer, Y. Peng, A. Thomson, H. Arora, R. Polski, Y. Zhang, H. Ren, J. Alicea, G. Refael et al., Nat. Phys. 15, 1174 (2019).

[16] M. S. Scheurer and R. Samajdar, Phys. Rev. Research 2, 033062 (2020).

[17] R. Samajdar and M. S. Scheurer, Phys. Rev. B 102, 064501 (2020).

[18] Y. Shimazaki, I. Schwartz, K. Watanabe, T. Taniguchi, M. Kroner, and A. Imamoğlu, Nature (London) 572, 215 (2019)
[19] S. Huang, K. Kim, D. K. Efimkin, T. Lovorn, T. Taniguchi, K. Watanabe, A. H. MacDonald, E. Tutuc, and B. J. LeRoy, Phys. Rev. Lett. 121, 037702 (2018).

[20] A. Kerelsky, C. Rubio-Verdú, L. Xian, D. M. Kennes, D. Halbertal, N. Finney, L. Song, S. Turkel, L. Wang, K. Watanabe et al., arXiv:1911.00007.

[21] C. Shen, Y. Chu, Q. Wu, N. Li, S. Wang, Y. Zhao, J. Tang, J. Liu, J. Tian, K. Watanabe et al., Nat. Phys. 16, 520 (2020).

[22] G. W. Burg, J. Zhu, T. Taniguchi, K. Watanabe, A. H. MacDonald, and E. Tutuc, Phys. Rev. Lett. 123, 197702 (2019).

[23] X. Liu, Z. Hao, E. Khalaf, J. Y. Lee, Y. Ronen, H. Yoo, D. H. Najafabadi, K. Watanabe, T. Taniguchi, A. Vishwanath et al., Nature (London) 583, 221 (2020).

[24] Y. Cao, D. Rodan-Legrain, O. Rubies-Bigorda, J. M. Park, K. Watanabe, T. Taniguchi, and P. Jarillo-Herrero, Nature (London) 583, 215 (2020).

[25] M. He, Y. Li, J. Cai, Y. Liu, K. Watanabe, T. Taniguchi, X. $\mathrm{Xu}$, and M. Yankowitz, Nat. Phys. (2020), doi: 10.1038/s41567020-1030-6.

[26] L. Wang, E. M. Shih, A. Ghiotto, L. Xian, D. A. Rhodes, C. Tan, M. Claassen, D. M. Kennes, Y. Bai et al., Nat. Mater. 19, 861 (2020).

[27] E. C. Regan, D. Wang, C. Jin, M. I. B. Utama, B. Gao, X. Wei, S. Zhao, W. Zhao, Z. Zhang, K. Yumigeta et al., Nature (London) 579, 359 (2020).

[28] Y. Tang, L. Li, T. Li, Y. Xu, S. Liu, K. Barmak, K. Watanabe, T. Taniguchi, A. H. MacDonald, J. Shan, and K. F. Mak, Nature (London) 579, 353 (2020).

[29] L. Xian, M. Claassen, D. Kiese, M. M. Scherer, S. Trebst, D. M. Kennes, and A. Rubio, arXiv:2004.02964.

[30] L. Xian, D. M. Kennes, N. Tancogne-Dejean, M. Altarelli, and A. Rubio, Nano Lett. 19, 4934 (2019).

[31] G. X. Ni, H. Wang, B.-Y. Jiang, L. X. Chen, Y. Du, Z. Y. Sun, M. D. Goldflam, A. J. Frenzel, X. M. Xie, M. M. Fogler, and D. N. Basov, Nat. Commun. 10, 4360 (2019).

[32] D. M. Kennes, L. Xian, M. Claassen, and A. Rubio, Nat. Commun. 11, 1124 (2020).

[33] D. M. Kennes, M. Claassen, L. Xian, A. Georges, A. J. Millis, J. Hone, C. R. Dean, D. N. Basov, A. Pasupathy, and A. Rubio, arXiv:2011.12638 [Nat. Phys. (to be published)].

[34] L. Klebl and C. Honerkamp, Phys. Rev. B 100, 155145 (2019).

[35] L. Klebl, D. M. Kennes, and C. Honerkamp, Phys. Rev. B 102, 085109 (2020).

[36] Y. Su and S.-Z. Lin, Phys. Rev. B 98, 195101 (2018).

[37] T. M. R. Wolf, J. L. Lado, G. Blatter, and O. Zilberberg, Phys. Rev. Lett. 123, 096802 (2019). 
[38] L. A. Gonzalez-Arraga, J. L. Lado, F. Guinea, and P. San-Jose, Phys. Rev. Lett. 119, 107201 (2017).

[39] M. Koshino, N. F. Q. Yuan, T. Koretsune, M. Ochi, K. Kuroki, and L. Fu, Phys. Rev. X 8, 031087 (2018).

[40] See Supplemental Material at http://link.aps.org/supplemental/ 10.1103/PhysRevB.103.L041103 for numerical details of the atomistic tight-binding model and RPA/mean-field calculations, including Refs. [34,39,46,47,52,56-62].

[41] H.-K. Tang, E. Laksono, J. N. B. Rodrigues, P. Sengupta, F. F. Assaad, and S. Adam, Phys. Rev. Lett. 115, 186602 (2015).

[42] D. S. de la Peña, J. Lichtenstein, C. Honerkamp, and M. M. Scherer, Phys. Rev. B 96, 205155 (2017).

[43] T. O. Wehling, E. Şaşioğlu, C. Friedrich, A. I. Lichtenstein, M. I. Katsnelson, and S. Blügel, Phys. Rev. Lett. 106, 236805 (2011).

[44] M. Rösner, E. Şaşioğlu, C. Friedrich, S. Blügel, and T. O. Wehling, Phys. Rev. B 92, 085102 (2015).

[45] M. Schüler, M. Rösner, T. O. Wehling, A. I. Lichtenstein, and M. I. Katsnelson, Phys. Rev. Lett. 111, 036601 (2013).

[46] N. Berk and J. Schrieffer, Phys. Rev. Lett. 17, 433 (1966).

[47] A. T. Rømer, S. Graser, T. S. Nunner, P. J. Hirschfeld, and B. M. Andersen, Phys. Rev. B 86, 054507 (2012).

[48] J. A. Hertz, Phys. Rev. B 14, 1165 (1976).
[49] D. Scalapino, Phys. Rep. 250, 329 (1995).

[50] A. M. Black-Schaffer and C. Honerkamp, J. Phys.: Condens. Matter 26, 423201 (2014).

[51] C. Honerkamp, Phys. Rev. Lett. 100, 146404 (2008).

[52] J.-X. Zhu, Bogoliubov-de Gennes Method and its Applications (Springer, New York, 2016), Vol. 924.

[53] X. Y. Xu, S. Wessel, and Z. Y. Meng, Phys. Rev. B 94, 115105 (2016).

[54] Y. Cao, D. Rodan-Legrain, J. M. Park, F. N. Yuan, K. Watanabe, T. Taniguchi, R. M. Fernandes, L. Fu, and P. Jarillo-Herrero, arXiv:2004.04148.

[55] M. W. Mitchell and S. Palacios Alvarez, Rev. Mod. Phys. 92, 021001 (2020).

[56] T. Ozaki, Phys. Rev. B 75, 035123 (2007).

[57] S. L. Cunningham, Phys. Rev. B 10, 4988 (1974).

[58] K. Uchida, S. Furuya, J.-I. Iwata, and A. Oshiyama, Phys. Rev. B 90, 155451 (2014).

[59] Z. Y. Rong and P. Kuiper, Phys. Rev. B 48, 17427 (1993).

[60] P. Moon and M. Koshino, Phys. Rev. B 87, 205404 (2013).

[61] J. M. B. Lopes dos Santos, N. M. R. Peres, and A. H. Castro Neto, Phys. Rev. B 86, 155449 (2012).

[62] A. O. Sboychakov, A. L. Rakhmanov, A. V. Rozhkov, and F. Nori, Phys. Rev. B 92, 075402 (2015). 